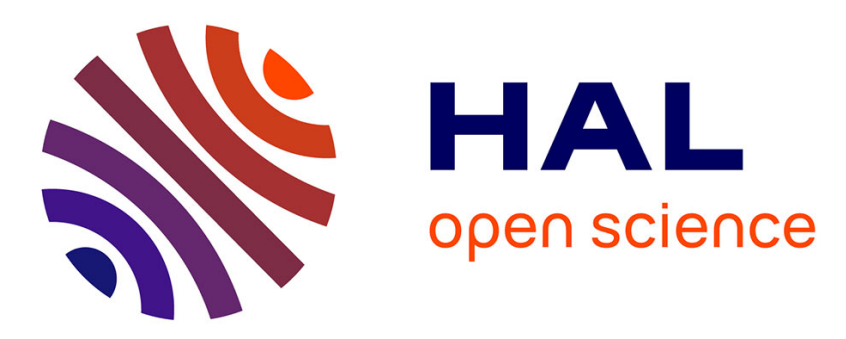

\title{
On Finite-Time Robust Stabilization via Nonlinear State Feedback
}

\author{
Konstantin Zimenko, Andrey Polyakov, Denis Efimov
}

\section{To cite this version:}

Konstantin Zimenko, Andrey Polyakov, Denis Efimov. On Finite-Time Robust Stabilization via Nonlinear State Feedback. International Journal of Robust and Nonlinear Control, 2018, 28 (16), pp.49514965. 10.1002/rnc.4292. hal-01830102

\section{HAL Id: hal-01830102 https://hal.inria.fr/hal-01830102}

Submitted on 4 Jul 2018

HAL is a multi-disciplinary open access archive for the deposit and dissemination of scientific research documents, whether they are published or not. The documents may come from teaching and research institutions in France or abroad, or from public or private research centers.
L'archive ouverte pluridisciplinaire HAL, est destinée au dépôt et à la diffusion de documents scientifiques de niveau recherche, publiés ou non, émanant des établissements d'enseignement et de recherche français ou étrangers, des laboratoires publics ou privés. 


\title{
On Finite-Time Robust Stabilization via Nonlinear State Feedback
}

\author{
K. Zimenko ${ }^{1}$, A. Polyakov ${ }^{1,2,3}$, D. Efimov ${ }^{1,2,3}$ \\ ${ }^{1}$ Department of Control Systems and Informatics, ITMO University, 49 Kronverkskiy av., 197101 Saint Petersburg, \\ Russia \\ 2 Non-A team, Inria Lille-Nord Europe, Parc Scientifique de la Haute Borne 40, avenue Halley Bat. A, Park Plaza, \\ 59650 Villeneuve d'Ascq, France \\ ${ }^{3}$ CRIStAL (UMR-CNRS 9189), Ecole Centrale de Lille, BP 48, Cite Scientifique, 59651 Villeneuve-d'Ascq, France
}

\begin{abstract}
SUMMARY
A nonlinear control law is designed for finite-time stabilization of a chain of integrators. The method is based on Implicit Lyapunov Function (ILF) technique and homogeneity properties. The scheme of control parameters selection is presented by a Linear Matrix Inequality (LMI). The method is simple in implementation and does not assume on-line procedure for computation of the ILF value at the current state that is an improvement with respect to [9], [13]. The control law is presented in an explicit form and allows to find the values of all control parameters, that make the solution one of the most constructive. The theoretical results are supported by numerical example.
\end{abstract}

Key words: Non-asymptotic stabilization; Implicit Lyapunov Function; Homogeneous systems

\section{INTRODUCTION}

Frequently, strict quality requirements are imposed for design, operation and control of complex technical processes. One such requirement is completion of all transients in a finite time. In particular, this issue is relevant in control design for a variety of robotic and mechatronic devices, safety evaluation, aerospace applications, vehicles control systems, etc. Therefore the problems of finite-time control and finite-time observation are intensively studied for many years (see, for example, [2]-[5] and [6]-[8], [22] correspondingly).

Solutions of the problem of control design for chain of integrators are quite sought-after. This is motivated by the fact that control scheme for chain of integrators can be easily extended to larger classes of systems (see, for example, [9]). Moreover, in many applications the nominal models have the form of chain of integrators, for instance, mechanical planar systems. There is a number of research results on the finite-time stabilization or regulation of a chain of integrators (see, for example, [10], [11]). However, most of them do not find an application in practice because of constructive and computational complexity. For instance, the methods mainly do not

Contract/grant sponsor: Russian Science Foundation; contract/grant number: 17-19-01422 
suggest constructive algorithms of parameter tuning and ensure only existence of suitable control parameters.

One of effective methods for the finite-time stability analysis is the method of Implicit Lyapunov Function (ILF) [9], [19], [20]. This method allows to check all stability conditions directly using implicitly defined Lyapunov function which is a solution of some algebraic equation. Note that for such a stability analysis there is no need to solve this equation. Another issue that can be useful in analysis of finite-time stability is property of homogeneity. For instance, if an asymptotically stable system is homogeneous of negative degree, then it is finite-time stable.

The paper [13] is related to development of method for finite-time control of multiple integrators together with implicit Lyapunov function of closed-loop system. Conditions of finite-time stability are formulated in the form of LMI that essentially simplifies the process of tuning of control parameters. However, the practical realization of the ILF control algorithm requires development of a special numeric procedure for on-line computation of the ILF value at the current state (the control law depends on ILF value explicitly). Thus, in the case of extension of this method on a class of technical systems, the need of additional on-line computations may be a stumbling block for implementation of this method.

The present paper addresses the problem of a control design for the finite-time stabilization of a chain of integrators. The paper represents an extension and simplification of results in [1], [13]. The developed finite-time control law does not depend on implicitly defined Lyapunov function that allows to avoid any additional on-line procedures. The result is obtained by using the method of Implicit Lyapunov Function with applying properties of homogeneous systems. As in [13] parameter tuning is presented in LMI form.

The paper is organized as follows. Notation used in the paper is given in the next section. Problem statement is considered in Section III. Some background notions about finite-time stability, ILF method and homogeneity are introduced in Section IV. Control design algorithm and some remarks about proposed control scheme are presented in Section V. Section VI contains simulation results for effectiveness demonstration of proposed control. Finally, concluding remarks and possible directions for future research are presented in Section VII.

\section{NOTATION}

Through the paper the following notation will be used:

(i) $\mathbb{R}_{+}=\{x \in \mathbb{R}: x>0\}$, where $\mathbb{R}$ is the set of real number;

(ii) $\|\cdot\|$ is the Euclidian norm in $\mathbb{R}^{n}$, i.e. $\|x\|=\sqrt{x_{1}^{2}+\cdots+x_{n}^{2}}$ for $x \in \mathbb{R}^{n}$;

(iii) the positive (negative) definiteness (semi-definiteness) of a symmetric matrix $P=P^{T} \in$ $\mathbb{R}^{n \times n}$ is denoted by $P>0(P<0, P \geq 0, P \leq 0)$;

(iv) for a symmetric matrix $P=P^{T}$ the minimal and maximal eigenvalues are denoted by $\lambda_{\min }(P)$ and $\lambda_{\max }(P)$, respectively;

(v) a continuous function $\sigma: \mathbb{R}_{+} \cup\{0\} \rightarrow \mathbb{R}_{+} \cup\{0\}$ belongs to the class $\mathcal{K}$ if $\sigma(0)=0$ and the function is strictly increasing;

(vi) $\operatorname{diag}\left\{\lambda_{i}\right\}_{i=1}^{n}$ is the diagonal matrix with the elements $\lambda_{i}$ on the main diagonal; 
(vii) for any $x \in \mathbb{R}^{n}$ the upper directional derivative $D V(x)$ of a locally Lipschitz continuous function $V: \mathbb{R}^{n} \rightarrow \mathbb{R}_{+}$in the direction of $\nu \in \mathbb{R}^{n}$ is defined as follows:

$$
D V(x) \nu=\limsup _{\substack{y \rightarrow x \\ t \rightarrow 0^{+}}} \frac{V(y+t \nu)-V(y)}{t},
$$

where lim sup denotes the upper limit.

\section{PROBLEM STATEMENT}

Consider the control system

$$
\dot{x}=A x+b u+d(t, x),
$$

where $x \in \mathbb{R}^{n}$ is the state vector, $u \in \mathbb{R}$ is the control input, $d(t, x): \mathbb{R}^{n+1} \rightarrow \mathbb{R}^{n}$ describes the system uncertainties and disturbances,

$$
A=\left(\begin{array}{ccccc}
0 & 1 & 0 & \cdots & 0 \\
0 & 0 & 1 & \cdots & 0 \\
\vdots & \vdots & \vdots & \ddots & \vdots \\
0 & 0 & 0 & \cdots & 1 \\
0 & 0 & 0 & \cdots & 0
\end{array}\right) \quad \text { and } \quad b=\left(\begin{array}{c}
0 \\
0 \\
\vdots \\
0 \\
1
\end{array}\right)
$$

which describes a chain of integrators for $d(t, x) \equiv 0$.

It is assumed that the disturbance $d(t, x)$ may be non-Lipschitz or discontinuous. This assumption makes presented system attractive for a number of control applications, for example, mechanical systems with dry friction, friction electromechanical system [26], cart-pendulum system [27], etc. Moreover, the controllable single-input nonlinear systems can be transformed to canonical form like (1) [28].

The aim of the paper is to develop the finite-time stabilizing control algorithm to the system (1), which is robust with respect to disturbances of certain class (to be defined below), has the form of the explicit full-state feedback (i.e. it does not require additional on-line computations as in [13]) and admits the simple LMI-based scheme for parameters tuning.

\section{PRELIMINARIES}

\subsection{Finite-time Stability}

Consider the system of the form

$$
\dot{x}=f(t, x), \quad x(0)=x_{0},
$$

where $x \in \mathbb{R}^{n}$ is the state vector, $f \in \mathbb{R}_{+} \times \mathbb{R}^{n} \rightarrow \mathbb{R}^{n}$ is a nonlinear vector field, which can be discontinuous with respect to the state variable. In this case the solutions $x\left(t, x_{0}\right)$ of the system (2) are understood in the sense of Filippov [14]. 
According to [14] an absolutely continuous function $x\left(t, x_{0}\right)$ is called a solution of the Cauchy problem associated to (2) if $x\left(0, x_{0}\right)=x_{0}$ and for almost all $t>0$ it satisfies the following differential inclusion

$$
\dot{x} \in K[f](t, x)=c o \bigcap_{\varepsilon>0} \bigcap_{\mu(N)=0} f(t, B(x, \varepsilon) \backslash N),
$$

where $c o(M)$ defines the convex closure of the set $M, B(x, \varepsilon)$ is the ball with the center at $x \in \mathbb{R}^{n}$ and the radius $\varepsilon$, the equality $\mu(N)=0$ means that the measure of $N \subset \mathbb{R}^{n}$ is zero. The solution existence is guaranteed due to the following multi-valued function properties (standart assumptions): the right-hand side of (3) has non-empty, compact, convex values and it is upper semi-continuous [29].

Assume that the origin is an equilibrium of the system (2) (i.e. $0 \in K[f](t, 0)$ ). Denote the set of all solutions of Cauchy problem (3) by $\Phi\left(x_{0}\right)$, i.e. $x\left(t, x_{0}\right) \in \Phi\left(x_{0}\right)$. The system (2) may have nonunique solutions for a given $x_{0} \in \mathbb{R}^{n}$ and may admit both weak (a property holds for a solution) and strong stability (a property holds for all solutions) (see, for example, [14], [3]). This paper deals only with the strong stability properties, which ask for stable behavior of all solutions of the system (2).

Definition 1 ([2], [3], [12], [26]):

The origin of system (2) is said to be globally finite-time stable if:

(i) Finite-time attractivity: there exists so-called settling time function $T: \mathbb{R}^{n} \backslash\{0\} \rightarrow \mathbb{R}_{+}$such that $\lim _{t \rightarrow T\left(x_{0}\right)} x\left(t, x_{0}\right)=0$ for all $x_{0} \in \mathbb{R}^{n} \backslash\{0\}$ and all $x\left(t, x_{0}\right) \in \Phi\left(x_{0}\right)$.

(ii) Lyapunov stability: there exists a function $\delta \in \mathcal{K}$ such that $\left\|x\left(t, x_{0}\right)\right\| \leq \delta\left(\left\|x_{0}\right\|\right)$ for all $x_{0} \in \mathbb{R}^{n}$ and all $x\left(t, x_{0}\right) \in \Phi\left(x_{0}\right)$.

\subsection{Homogeneity}

Homogeneity [15]-[17] is an intrinsic property whereby objects such as functions or vector fields remain consistent with respect to some scaling operation called a dilation.

For fixed $r_{i} \in \mathbb{R}_{+}, i \in\{1, \ldots, n\}$ called weights and $\lambda>0$ one can define:

(i) the vector of weights $r=\left(r_{1}, \ldots, r_{n}\right)^{T}$;

(ii) the dilation matrix

$$
D(\lambda)=\operatorname{diag}\left\{\lambda^{r_{i}}\right\}_{i=1}^{n}
$$

and correspondingly $D(\lambda) x=\left(\lambda^{r_{1}} x_{1}, \ldots, \lambda^{r_{n}} x_{n}\right)^{T}$ for $x=\left(x_{1}, \ldots, x_{n}\right)^{T} \in \mathbb{R}^{n}$.

Definition 2 [17]:

A function $g: \mathbb{R}^{n} \rightarrow \mathbb{R}$ (vector field $f: \mathbb{R}^{n} \rightarrow \mathbb{R}^{n}$ ) is said to be $r$-homogeneous of degree $m$ iff $g(D(\lambda) x)=\lambda^{m} g(x)\left(f(D(\lambda) x)=\lambda^{m} D(\lambda) f(x)\right)$ for all $\lambda>0$ and $x \in \mathbb{R}^{n}$.

Let us introduce the homogeneous norm $\|x\|_{r}$ of $x \in \mathbb{R}^{n}$ defined by

$$
\|x\|_{r}=\left(\sum_{i=1}^{n}\left|x_{i}\right|^{\frac{\rho}{r_{i}}}\right)^{\frac{1}{\rho}}
$$

for $\rho \geq \max _{i} r_{i}$. Note, that homogeneous norm (4) is not a norm in classical sense, since the triangular inequality is not satisfied. 
Obviously, each homogeneous norm is $r$-homogeneous of degree 1 and positive definite. For any $x \in \mathbb{R}^{n}$ there are $y \in S_{r}=\left\{x \in \mathbb{R}^{n}:\|x\|_{r}=1\right\}$ and $\lambda=\|x\|_{r}$ such that $x=D(\lambda) y[18]$.

Theorem 1 [18]

Let $f$ be a $r$-homogeneous of degree $m$ continuous vector field on $\mathbb{R}^{n}$ such that the system (2) is a locally asymptotically stable. Then it is globally asymptotically stable and there exists a continuously differentiable $r$-homogeneous of degree $\varpi>-m$ Lyapunov function $V$ for (2).

Note that by definition of homogeneity there exist constants $c_{1}, c_{2}>0$ such that

$$
c_{1}\|x\|_{r}^{\varpi} \leq V(x) \leq c_{2}\|x\|_{r}^{\varpi} .
$$

Theorem 2 [18]

Let $f: \mathbb{R}^{n} \rightarrow \mathbb{R}^{n}$ be defined on $\mathbb{R}^{n}$ and be a continuous $r$-homogeneous vector field with a negative degree. If the origin of the system (2) is locally attractive then it is globally finite time stable.

The following theorem and corollary extend presented results to differential inclusions.

\section{Definition 3}

A set-valued map $F: \mathbb{R}^{n} \rightrightarrows \mathbb{R}^{n}$ is said to be r-homogeneous of degree $m \in \mathbb{R}$ if for all $x \in \mathbb{R}^{n}$ we have

$$
F(D(\lambda) x)=\lambda^{m} D(\lambda) F(x), \quad \forall x \in \mathbb{R}^{n} \backslash\{0\}, \quad \forall \lambda \in \mathbb{R}_{+} .
$$

The system

$$
\dot{x} \in F(x), \quad x(0)=x_{0} \in \mathbb{R}^{n},
$$

is $r$-homogeneous of degree $m$ if the set-valued map $F$ is homogeneous of degree $m$.

Theorem 3 [30]

Let $F$ be a $r$-homogeneous set-valued map with degree $m$, satisfying the standard assumptions. Then the next claims are equivalent:

1. The system (7) is strongly globally asymptotically stable.

2. For all $k>\max (-m, 0)$, there exist a pair $(V, W)$ of continuous functions, such that: $V \in$ $\mathcal{C}^{\infty}\left(\mathbb{R}^{n}\right)$ is positive definite and homogeneous with degree $k ; W \in \mathcal{C}^{\infty}\left(\mathbb{R}^{n} \backslash\{0\}\right)$ is strictly positive outside the origin and homogeneous with degree $k+m ; \max _{\nu \in F(x)} D V(x) \nu \leq$ $-W(x)$ for all $x \neq 0$.

\section{Corollary 1 [30]}

Let $F$ be a $r$-homogeneous set-valued map with degree $m<0$, satisfying the standard assumptions. Assume also that $F$ is strongly globally asymptotically stable. Then $F$ is strongly globally finite-time stable and the settling-time function is continuous at zero and locally bounded.

\subsection{Implicit Lyapunov Function Method}

The theorem given below presents the ILF method ([19], [20]) for finite-time stability analysis. 
Theorem 4 [9], [13]

If there exists a continuous function

$$
\begin{aligned}
& Q: \mathbb{R}_{+} \times \mathbb{R}^{n} \rightarrow \mathbb{R} \\
& (V, x) \mapsto Q(V, x)
\end{aligned}
$$

such that

C1) $Q(V, x)$ is continuously differentiable for $\forall x \in \mathbb{R}^{n} \backslash\{0\}$ and $\forall V \in \mathbb{R}_{+}$;

C2) for any $x \in \mathbb{R}^{n} \backslash\{0\}$ there exist $V^{-} \in \mathbb{R}_{+}$and $V^{+} \in \mathbb{R}_{+}$:

$$
Q\left(V^{-}, x\right)<0<Q\left(V^{+}, x\right)
$$

C3) for $\Omega=\left\{(V, x) \in \mathbb{R}^{n+1}: Q(V, x)=0\right\}$

$$
\lim _{\substack{x \rightarrow 0 \\(V, x) \in \Omega}} V=0^{+}, \lim _{\substack{V \rightarrow 0^{+} \\(V, x) \in \Omega}}\|x\|=0, \lim _{\substack{\|x\| \rightarrow \infty \\(V, x) \in \Omega}} V=+\infty
$$

C4) the inequality

$$
-\infty<\frac{\partial Q(V, x)}{\partial V}<0
$$

holds for $\forall V \in \mathbb{R}_{+}$and $\forall x \in \mathbb{R}^{n} \backslash\{0\}$;

C5) the inequality

$$
\frac{\partial Q(V, x)}{\partial x} f(x) \leq \eta V^{1-\mu} \frac{\partial Q(V, x)}{\partial V}
$$

holds $\forall(V, x) \in \Omega$, where $0<\mu \leq 1$ and $\eta>0$ are some constants.

Then the origin of the system (2) is globally finite-time stable with the following settling time estimate

$$
T\left(x_{0}\right) \leq \frac{V_{0}^{\mu}}{\eta \mu}
$$

where $V_{0} \in \mathbb{R}_{+}: Q\left(V_{0}, x_{0}\right)=0$.

The conditions of Theorem 4 present in an implicit form the requirements on finite-time stability [5]. The conditions $C 1, C 2, C 4$ and the implicit function theorem [31] imply that the equation $Q(V, x)=0$ implicitly defines a unique function $V: \mathbb{R}^{n} \backslash\{0\} \rightarrow \mathbb{R}_{+}$such that $Q(V(x), x)=0$ for all $x \in \mathbb{R}^{n} \backslash\{0\}$. Due to the condition $C 3$ the function $V$ can be continuously prolonged at the origin by setting $V(0)=0$. In addition, it is radially unbounded and positive definite. In conjunction with the condition $C 5$, Theorem 4 guarantees fulfillment of sufficient conditions of finite-time stability mentioned, for example, in [2], [5].

\section{MAIN RESULT}

Introduce the ILF function

$$
Q(V, x):=x^{T} D\left(V^{-1}\right) P D\left(V^{-1}\right) x-1,
$$


where $P=P^{T} \in \mathbb{R}^{n \times n}$ is a symmetric positive definite matrix, $D(\lambda)$ is the dilation matrix of the form

$$
D(\lambda)=\operatorname{diag}\left\{\lambda^{1+(n-i) \mu}\right\}
$$

for $0<\mu \leq 1$.

Note that for $r=(1+(n-1) \mu, 1+(n-2) \mu, \ldots, 1)^{T}$ the Lyapunov function $V(x)$ is $r$ homogeneous of degree 1 as $Q(V, D(\lambda) x)=Q\left(\lambda^{-1} V, x\right)$, i.e. $V(D(\lambda) x)=\lambda V(x)$ and the inequality (5) for $\varpi=1$ takes the form

$$
c_{1}\|x\|_{r} \leq V(x) \leq c_{2}\|x\|_{r} .
$$

Let us denote the diagonal matrices $H_{\mu}=\operatorname{diag}\{1+(n-i) \mu\}_{i=1}^{n}, \quad H(\lambda)=$ $\operatorname{diag}\left\{\lambda^{(n+1-i) \mu}\right\}_{i=1}^{n}$.

Proposition 1

If the following statements hold:

1) the system of matrix inequalities

$$
\begin{gathered}
\left(\begin{array}{cc}
A X+X A^{T}+b y+y^{T} b^{T}+L & b y \\
y^{T} b^{T} & -\gamma X
\end{array}\right)<0, \\
\frac{1}{\nu} L \geq X H_{\mu}+H_{\mu} X>0, \quad X>0, \\
Z \leq \frac{1}{\gamma} X, \quad Z>0, \\
\left(\begin{array}{cc}
\beta L & X(I-H(\theta)) \\
(I-H(\theta)) X & Z
\end{array}\right) \geq 0, \quad \forall \theta \in\left[\theta_{\min }, \theta_{\max }\right]
\end{gathered}
$$

is feasible for some $\mu \in(0,1], \beta \in(0,1), \gamma, \nu, \theta_{\min }, \theta_{\max } \in \mathbb{R}_{+}, L, Z, X \in \mathbb{R}^{n \times n}, y \in \mathbb{R}^{1 \times n}$;

2) there exists a $c_{u} \in \mathbb{R}_{+}$that satisfies the inequality

$$
\frac{c_{2}}{\theta_{\max }} \leq c_{u} \leq \frac{c_{1}}{\theta_{\min }}
$$

where the coefficients $c_{1}$ and $c_{2}$ correspond to the inequality (5).

Then the control of the form

$$
u(x)=\left(c_{u}\|x\|_{r}\right)^{1-\mu} k D\left(\left(c_{u}\|x\|_{r}\right)^{-1}\right) x,
$$

where $k=y X^{-1}$ stabilizes the origin of the system (1) in a finite time for the case $d(t, x)=0$ and the settling time function is bounded as follows

$$
T\left(x_{0}\right) \leq \frac{c_{2}^{\mu}\left\|x_{0}\right\|_{r}^{\mu}}{\nu \mu(1-\beta)} .
$$


Proof of Proposition 1 According to [9] the system (1) is finite-time robustly stable with some disturbance function $d(t, x)$ if:

1) the following system of inequalities is feasible

$$
\left\{\begin{array}{l}
A X+X A^{T}+b y+y^{T} b^{T}+L+R \leq 0 \\
\frac{1}{\nu} L \geq X H_{\mu}+H_{\mu} X>0, \quad X>0
\end{array}\right.
$$

for some $\mu \in(0,1], \nu \in \mathbb{R}_{+}$and $R \in \mathbb{R}^{n \times n}, R>0$;

2) the conrol has the form

$$
u(V, x)=V^{1-\mu} k D\left(V^{-1}\right) x,
$$

where $V \in \mathbb{R}_{+}: Q(V, x)=0$ and $Q(V, x)$ presented by (9) with $P=X^{-1}$;

$3)$ the disturbance function $d(t, x)$ satisfies the following inequality

$$
d^{T}(t, x) D\left(V^{-1}\right) R^{-1} D\left(V^{-1}\right) d(t, x) \leq \beta V^{-2 \mu} x^{T} D\left(V^{-1}\right) P L P D\left(V^{-1}\right) x
$$

for some $\beta \in(0,1)$.

The settling time function estimate has the form

$$
T\left(x_{0}\right) \leq \frac{V_{0}^{\mu}}{\nu \mu(1-\beta)} .
$$

All proofs of function $Q(V, x)$ compliance with the conditions $C 1)-C 5$ ) can be found in [9].

Let us substitute $c_{u}\|x\|_{r}$ for the function $V(x)$ in (19) and represent difference of controls as a disturbance in the equation $\dot{x}=A x+b u+d(t, x)$ :

$$
\begin{aligned}
& d(t, x)=-b\left(V^{1-\mu} k D\left(V^{-1}\right)-\left(c_{u}\|x\|_{r}\right)^{1-\mu} k D\left(\left(c_{u}\|x\|_{r}\right)^{-1}\right)\right) x= \\
& -V^{1-\mu} b k\left(I_{n}-H\left(\frac{V}{c_{u}\|x\|_{r}}\right)\right) D\left(V^{-1}\right) x .
\end{aligned}
$$

Choose the matrix $R$ in the form

$$
R=\left(\begin{array}{cc}
\varrho I_{n-1} & 0 \\
0 & r_{n n}
\end{array}\right),
$$

where $\varrho, r_{n n} \in \mathbb{R}_{+}$, $\varrho$ can be arbitrarily small. Then, substituting (22) into (20) and taking into account that $b^{T} D\left(V^{-1}\right) R^{-1} D\left(V^{-1}\right) b=V^{-2} r_{n n}^{-1}$ it is easy to obtain:

$$
\begin{gathered}
V^{2-2 \mu} x^{T} D\left(V^{-1}\right)\left(I_{n}-H\left(\frac{V}{c_{u}\|x\|_{r}}\right)\right) k^{T} b^{T} D\left(V^{-1}\right) R^{-1} D\left(V^{-1}\right) b k\left(I_{n}-H\left(\frac{V}{c_{u}\|x\|_{r}}\right)\right) D\left(V^{-1}\right) x \leq \\
\beta V^{-2 \mu} x^{T} D\left(V^{-1}\right) P L P D\left(V^{-1}\right) x \\
\frac{1}{r_{n n}} x^{T} D\left(V^{-1}\right)\left(I_{n}-H\left(\frac{V}{c_{u}\|x\|_{r}}\right)\right) k^{T} k\left(I_{n}-H\left(\frac{V}{c_{u}\|x\|_{r}}\right)\right) D\left(V^{-1}\right) x \leq \beta x^{T} D\left(V^{-1}\right) P L P D\left(V^{-1}\right) x \\
\Downarrow \\
\frac{1}{r_{n n}}\left(I-H\left(\frac{V}{c_{u}\|x\|_{r}}\right)\right) k^{T} k\left(I-H\left(\frac{V}{c_{u}\|x\|_{r}}\right)\right) \leq \beta P L P .
\end{gathered}
$$


Let $r_{n n}=\frac{1}{\gamma} k P^{-1} k^{T}$. In this case $R=r_{n n} b b^{T}+\left(\begin{array}{rr}\varrho I_{n-1} & 0 \\ 0 & 0\end{array}\right)=\frac{1}{\gamma} b y X^{-1} y^{T} b^{T}+\left(\begin{array}{rr}\varrho I_{n-1} & 0 \\ 0 & 0\end{array}\right)$ and the first inequality in (18) is equivalent to the inequality

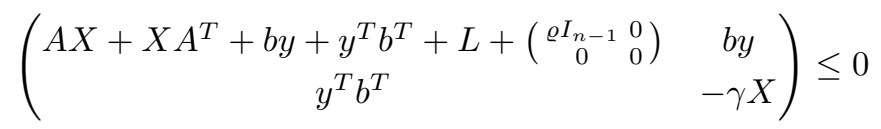

(for given $R$ it can be obtained from the first inequality in (18) by using Schur complement). Since $\varrho$ can be chosen arbitrary small this inequality can be replaced by strong inequality (11).

Since

$$
\frac{1}{r_{n n}} P^{-1 / 2} k^{T} k P^{-1 / 2}=\gamma \frac{P^{-1 / 2} k^{T} k P^{-1 / 2}}{k P^{-1} k^{T}}=\gamma \frac{s^{T} s}{s s^{T}} \leq \gamma I_{n} \Longleftrightarrow \frac{1}{r_{n n}} k^{T} k \leq \gamma P,
$$

where $s=k P^{-1 / 2}$, then introducing the inequality $\gamma P \leq Z^{-1}$ (equivalent to (13) with $P=X^{-1}$ ) we yield

$$
\left(I-H\left(\frac{V}{c_{u}\|x\|_{r}}\right)\right) Z^{-1}\left(I-H\left(\frac{V}{c_{u}\|x\|_{r}}\right)\right) \leq \beta P L P .
$$

Let $\theta=\frac{V}{c_{u}\|x\|_{r}}$, where $\theta \in\left[\theta_{\min }, \theta_{\max }\right]$. Then, using Schur complement and multiplying both sides of obtained expression by $\operatorname{diag}\left\{P^{-1}, I\right\}$ the inequality (14) can be derived. Thus, both conditions (18), (20) are satisfied.

On the other hand, according to (10) $\theta \in\left[\frac{c_{1}}{c_{u}}, \frac{c_{2}}{c_{u}}\right]$ and the parameter $c_{u}$ should satisfy to the inequality (15).

Finally, the settling time function can be estimated as follows

$$
T\left(x_{0}\right) \leq \frac{V_{0}^{\mu}}{\nu \mu(1-\beta)} \leq \frac{c_{2}^{\mu}\left\|x_{0}\right\|_{r}^{\mu}}{\nu \mu(1-\beta)},
$$

where $V_{0} \in \mathbb{R}_{+}: Q\left(V_{0}, x_{0}\right)=0$.

In order to apply Proposition 1 in practice we need to solve the parametrized system of nonlinear matrix inequalities (11)-(14) with respect to variables $X, y, Z, L, \theta_{\min }$ and $\theta_{\max }$ for a given $\gamma, \nu \in \mathbb{R}_{+}, \mu \in(0,1]$, and $\beta \in(0,1)$. By fixing the value $\theta \in\left[\theta_{\min }, \theta_{\max }\right]$, the system (11)-(14) becomes a system of LMIs, which can be solved using any suitable software (e.g. MATLAB, YALMIP, etc.). However, the LMIs have to be checked for any $\theta \in\left[\theta_{\min }, \theta_{\max }\right]$. Due to the smoothness of $H(\theta)$ with respect to $\theta \in \mathbb{R}_{+}$, this can be done on a proper grid constructed over the interval $\left[\theta_{\min }, \theta_{\max }\right]$. The following proposition provides sufficient feasibility condition of the inequality (14).

Proposition 2

The parametric matrix inequality (14) holds if for some $\mu \in(0,1], \beta \in(0,1)$ and $\theta_{\min }, \theta_{\max } \in \mathbb{R}_{+}$

$$
\begin{gathered}
\left(\begin{array}{ccc}
\beta L & X \tilde{H} & X\left(I-H\left(\theta_{i}\right)\right) \\
\tilde{H} X & \frac{1}{\left(\theta_{i}-\theta_{i-1}\right)} \tilde{M} & 0 \\
\left(I-H\left(\theta_{i}\right)\right) X & 0 & Z
\end{array}\right) \geq 0, \quad i=1, \ldots, N, \\
Z \tilde{H}+\tilde{H} Z \geq \tilde{M}, \quad \tilde{M}>0,
\end{gathered}
$$


where $\theta_{\min }=\theta_{0}<\theta_{1}<\cdots<\theta_{N}=\theta_{\max }, \tilde{H}=\operatorname{diag}\{(n+1-i) \mu\}_{i=1}^{n}$.

Proof of Proposition 2 Taking into account the left-hand side of the inequality (23) let us define $W(\theta)=z^{T}(I-H(\theta)) Z^{-1}(I-H(\theta)) z$ for some $z \in \mathbb{R}^{n}$, then

$$
\frac{\partial W}{\partial \theta}=\left(\begin{array}{cc}
(I-H(\theta)) z \\
\frac{1}{\theta} z
\end{array}\right)^{T}\left(\begin{array}{cc}
\frac{1}{\theta}\left(\tilde{H} Z^{-1}+Z^{-1} \tilde{H}\right) & -Z^{-1} \\
-Z^{-1} & \theta^{2} M^{-1}
\end{array}\right)\left(\begin{array}{c}
(I-H(\theta)) z \\
\frac{1}{\theta} z
\end{array}\right)-z^{T} \tilde{H} M^{-1} \tilde{H} z,
$$

for some $M>0$.

The matrix inequality $\left(\begin{array}{cc}\frac{1}{\theta}\left(\tilde{H} Z^{-1}+Z^{-1} \tilde{H}\right) & -Z^{-1} \\ -Z^{-1} & \theta^{2} M^{-1}\end{array}\right)>0$ is equivalent to

$$
\tilde{H} Z+Z \tilde{H}-\frac{1}{\theta} M>0
$$

that can be shown by using Schur complement and multiplying both sides by $Z$. Since $\theta \geq \frac{c_{1}}{c_{u}}$ the inequality $\tilde{M}=\frac{c_{u}}{c_{1}} M \geq \frac{1}{\theta} M>0$ holds. Then, according to (25) the inequality (26) holds.

In this case $\frac{\partial W}{\partial \theta} \geq-z^{T} \tilde{H} M^{-1} \tilde{H} z$. Integrating this inequality on interval $\left[\theta_{i-1}, \theta_{i}\right]$ we obtain

$$
W\left(\theta_{i-1}\right) \leq W\left(\theta_{i}\right)+\left(\theta_{i}-\theta_{i-1}\right) z^{T} \tilde{H} M^{-1} \tilde{H} z
$$

or according to (23)

$$
\left(I-H\left(\theta_{i}\right)\right) Z^{-1}\left(I-H\left(\theta_{i}\right)\right)+\left(\theta_{i}-\theta_{i-1}\right) \tilde{H} M^{-1} \tilde{H} \leq \beta P L P .
$$

Finally, using Schur complement and multiplying both sides of obtained matrix by $\operatorname{diag}\left\{P^{-1}, I, I\right\}$ obtain the following inequality

$$
\left(\begin{array}{ccc}
\beta L & X \tilde{H} & X\left(I-H\left(\theta_{i}\right)\right) \\
\tilde{H} X & \frac{c_{1}}{c_{u}}\left(\theta_{i}-\theta_{i-1}\right)^{-1} \tilde{M} & 0 \\
\left(I-H\left(\theta_{i}\right)\right) X & 0 & Z
\end{array}\right) \geq 0 .
$$

The value of $\frac{c_{1}}{c_{u}}$ for simplicity can be chosen equal to 1 since it affects only on number $N$ and size of the sampling step $\left(\theta_{i}-\theta_{i-1}\right)$. Thus, if inequalities (24), (25) are satisfied for all sampling steps $\left(\theta_{i}-\theta_{i-1}\right), i=1, \ldots, N$, the inequality (14) holds.

Proposition 2 allows to solve the parametrized system of matrix inequalities (11)-(14) using the following algorithm with fixed $\mu, \theta_{\min }$ and $\theta_{\max }$.

Algorithm 1

Initialization: $p=0, N=1, \theta_{0}=\theta_{\min }, \theta_{N}=\theta_{\max }, \sum_{0}=\left\{\theta_{0}, \theta_{N}\right\}$.

Loop: While the system of LMIs (11), (12), (13), (24), (25) with $\theta_{i} \in \sum_{p}$ is not feasible, do $\sum_{p+1}=\sum_{p} \cup\left\{\frac{\theta_{i-1}+\theta_{i}}{2}\right\}_{i=1}^{N}$ with $\theta_{i} \in \sum_{p}, N \leftarrow 2 N$ and $p \leftarrow p+1$.

For any fixed $\mu, \nu, \gamma \in \mathbb{R}_{+}$the inequalities (11), (12) are feasible at least for sufficiently small $\mu \in(0,1]$ and $\nu \in \mathbb{R}_{+}$[13]. Moreover, it is obvious that (13), (25) are feasible and since for $\mu \rightarrow 0$ : $(I-H(\theta)) \rightarrow 0$, then the presented algorithm always finds the required solution for sufficiently small $\mu$. Thus, one can always find such $\mu$ for which the system (1) is finite-time stable with control in the form (16). 
Since in addition to solving the system of LMIs (11)-(13), (24), (25) it is necessary to satisfy the inequality (15), a particular attention should be paid to selecting the values of the parameters $\theta_{\min }, \theta_{\max }$. Taking into account that according to (22) $d(t, x)=0$ for $\theta=1$ it is preferable to choose $0<\theta_{\min }<1$ and $\theta_{\max }>1$ to satisfy the inequality (14). On the other hand, to fulfil the inequality (15) it is necessary to solve the inequalities (11)-(14) with sufficiently large value of $\frac{\theta_{\max }}{\theta_{\min }}$. Thus, to select the parameters, the following strategy can be used along with Algorithm 1: starting with $\theta_{\max }=\theta_{\min }=1$ it is necessary to solve the system of inequalities (11)-(13), (24), (25) using Algorithm 1 with slightly increasing the parameter $\theta_{\max }$ and/or decreasing $\theta_{\min }$ until the inequality $\frac{c_{2}}{\theta_{\max }} \leq \frac{c_{1}}{\theta_{\min }}$ is satisfied. Then the parameter $c_{u}$ should be chosen according to (15).

Let us make some remarks about presented control scheme:

(i) For practical implementation of the control (16) feasibility of the inequality (15) is required. Therefore, it is necessary to estimate the coefficients $c_{1}$ and $c_{2}$.

To this end consider the set $D=\left\{x \in \mathbb{R}^{n} \mid V(x)=1\right\}$. Since $V \in \mathbb{R}_{+}: Q(V, x)=0$ for the set $D$ one can obtain $x^{T} P x=1$ and thus the following inequality holds

$$
\frac{1}{\lambda_{\max }(P)} \leq \sum_{i=1}^{n}\left|x_{i}\right|^{2} \leq \frac{1}{\lambda_{\min }(P)} \text {. }
$$

Hence

$$
\left|x_{i}\right| \leq \frac{1}{\lambda_{\min }^{\frac{1}{2}}(P)} \text {. }
$$

For $P \geq I\left(\lambda_{\min }(P) \geq 1\right)$ we have

$$
\|x\|_{r}=\left(\sum_{i=1}^{n}\left|x_{i}\right|^{\frac{\rho}{r_{i}}}\right)^{\frac{1}{\rho}} \leq\left(\sum_{i=1}^{n} \frac{1}{\lambda_{\min }^{\frac{\rho}{2 r_{i}}}(P)}\right)^{\frac{1}{\rho}} \leq\left(\frac{n}{\lambda_{\min }^{\frac{\rho}{2 r_{\max }}}(P)}\right)^{\frac{1}{\rho}}
$$

and for $P \nsupseteq I\left(\lambda_{\min }(P)<1\right)$

$$
\|x\|_{r}=\left(\sum_{i=1}^{n}\left|x_{i}\right|^{\frac{\rho}{r_{i}}}\right)^{\frac{1}{\rho}} \leq\left(\sum_{i=1}^{n} \frac{1}{\lambda_{\min }^{\frac{\rho}{2 r_{i}}}(P)}\right)^{\frac{1}{\rho}} \leq\left(\frac{n}{\lambda_{\min }^{\frac{\rho}{2 r_{\min }}}(P)}\right)^{\frac{1}{\rho}} .
$$

On the other hand, rewrite (10) for the set $D$ :

$$
c_{1}\|x\|_{r} \leq 1 \leq c_{2}\|x\|_{r} .
$$

Then basing on (29), (30) the estimation of $c_{1}$ can be taken as follows

$$
c_{1}=\min \left\{\frac{\lambda_{\min }^{\frac{1}{2 r_{\max }}}(P)}{n^{\frac{1}{\rho}}}, \frac{\lambda_{\min }^{\frac{1}{2 r_{\min }}}(P)}{n^{\frac{1}{\rho}}}\right\} .
$$

For estimation of $c_{2}$ one can obtain

$$
\frac{1}{\lambda_{\max }(P)} \leq \sum_{i=1}^{n}\left|x_{i}\right|^{2} \leq \sum_{i=1}^{n}\|x\|_{r}^{2 r_{i}} \leq n \begin{cases}\|x\|_{r}^{2 r_{\min }}, & \text { if }\|x\|_{r}<1 \\ \|x\|_{r}^{2 r_{\max }}, & \text { if }\|x\|_{r} \geq 1\end{cases}
$$


and basing on (31) the following estimation of $c_{2}$ can be taken

$$
c_{2}=\max \left\{\left(n \lambda_{\max }(P)\right)^{\frac{1}{2 r_{\max }}},\left(n \lambda_{\max }(P)\right)^{\frac{1}{2 r_{\min }}}\right\} .
$$

To get better estimations of $c_{1}$ and $c_{2}$, which is needed for relaxing the inequality (15), the following procedure can be applied. Let us denote the diagonal matrix $J=\operatorname{diag}\left\{\frac{1}{\sqrt{P_{i i}}}\right\}_{i=1}^{n}$. Rewrite (9) and (31) in the next forms

$$
\begin{gathered}
Q\left(V, x_{J}\right):=x_{J}^{T} D\left(V^{-1}\right) \tilde{P} D\left(V^{-1}\right) x_{J}-1, \\
c_{1}\left\|x_{J}\right\|_{r} \leq 1 \leq c_{2}\left\|x_{J}\right\|_{r},
\end{gathered}
$$

where $x_{J}=J^{-1} x$ and $\tilde{P}=J P J$. Note that the matrix $\tilde{P}$ has the values 1 on the main diagonal and eigenvalues of $\tilde{P}$ are close to 1 (i.e. $\lambda_{\min }(\tilde{P}) \leq 1$ and $\left.\lambda_{\max }(\tilde{P}) \geq 1\right)$. In this case we can obtain the estimations

$$
c_{1}=\frac{\lambda_{\min }^{\frac{1}{2 r_{\min }}}(\tilde{P})}{n^{\frac{1}{\rho}}}
$$

and

$$
c_{2}=\left(n \lambda_{\max }(\tilde{P})\right)^{\frac{1}{2 r_{\min }}},
$$

which in most cases are located closer to each other (and closer to the value 1) in comparison with (32), (33). This issue allows the inequality (15) to be relaxed.

(ii) The parameters $\beta$ and $\nu$ are tuning parameters for the upperbound of the settling time of the closed-loop system (see, the estimate (17)).

(iii) The control (16) is bounded with $|u(x)| \leq\left(c_{u}\left\|x_{0}\right\|_{r}\right)^{1-\mu} \sqrt{\xi \sum_{i=1}^{n} c_{u}^{-2 r_{i}}}$, where $\xi \in$ $\mathbb{R}_{+}: k^{T} k \leq \xi I$ (can be rewritten in LMI form as $\left(\begin{array}{cc}X & y^{T} \\ y & \xi_{1}\end{array}\right) \geq 0, \xi_{2} I \leq X$, where $\xi=\frac{\xi_{1}}{\xi_{2}}$ ). Indeed,

$$
\begin{aligned}
& u^{2}(x)=\left(c_{u}\|x\|_{r}\right)^{2-2 \mu} x^{T} D\left(\left(c_{u}\|x\|_{r}\right)^{-1}\right) k^{T} k D\left(\left(c_{u}\|x\|_{r}\right)^{-1}\right) x \leq \\
& \xi\left(c_{u}\|x\|_{r}\right)^{2-2 \mu} x^{T} D^{2}\left(\left(c_{u}\|x\|_{r}\right)^{-1}\right) x \leq \\
& \xi\left(c_{u}\left\|x_{0}\right\|_{r}\right)^{2-2 \mu}\left(\begin{array}{llll}
1 & 1 & \cdots & 1
\end{array}\right) D^{2}\left(c_{u}^{-1}\right)\left(\begin{array}{llll}
1 & 1 & \cdots & 1
\end{array}\right)^{T}=\xi\left(c_{u}\left\|x_{0}\right\|_{r}\right)^{2-2 \mu} \sum_{i=1}^{n} c_{u}^{-2 r_{i}}
\end{aligned}
$$

It is easy to see, if $\mu=1$ then the control (16) is globally bounded by $\sqrt{\xi \sum_{i=1}^{n} c_{u}^{-2 r_{i}}}$.

For $\mu \in(0,1)$ the control (16) is continuous in the state variable $x$. If $\mu \rightarrow 0$ then the feedback (16) becomes a linear $u=k x$. If $\mu=1$ the control is discontinuous at the origin and continuous outside. For practical realization of the control, the discontinuous feedback law can be replaced with a high-gain linear feedback if the system state is close to the origin as in [13].

(iv) The presented analysis can be performed for other control laws where instead of the homogeneous norm in (16) another positive homogeneous function of degree 1 is used. Search of such functions, for which the inequalities (14), (15) become less conservative, may be one of the directions of future research. 
(v) In comparison with the results of the work [13] the control (16) does not require any additional computational power for on-line computation of the ILF value at the current state. This issue significantly simplifies the practical realization of the developed finite-time control scheme.

The system (1) with the control (16) is $r$-homogeneous of degree $-\mu$ with the vector of weights $r=(1+(n-1) \mu, 1+(n-2) \mu, \ldots, 1)^{T}$. Indeed, since homogeneous norm is $r$-homogeneous of degree 1, i.e. $\|D(\lambda) x\|_{r}=\lambda\|x\|_{r}$ :

$$
\begin{aligned}
& u(D(\lambda) x)=\left(c_{u}\|D(\lambda) x\|_{r}\right)^{1-\mu} k D\left(\left(c_{u}\|D(\lambda) x\|_{r}\right)^{-1}\right) D(\lambda) x= \\
& \lambda^{1-\mu}\|x\|_{r} k D\left(\lambda^{-1}\left(c_{u}\|x\|_{r}\right)^{-1}\right) D(\lambda) x=\lambda^{1-\mu} u(x)
\end{aligned}
$$

and $A D(\lambda) x+B u(D(\lambda) x)=\lambda^{-\mu} D(\lambda)(A x+B u(x))$. Thus, following [21] the closed-loop system is robust with respect to measurement noises and exogenous perturbations.

If the system (1) has additional disturbances $d$, then obtained result can be easily extended.

Theorem 5

If the following statements hold:

1) the system of matrix inequalities (12), (13) is feasible with

$$
\begin{gathered}
\left(\begin{array}{cc}
\epsilon\left(A X+X A^{T}+b y+y^{T} b^{T}\right)+L_{1} & b y \\
y^{T} b^{T} & -\gamma X
\end{array}\right)<0, \\
\left(\begin{array}{cc}
\beta_{1} L & X(I-H(\theta)) \\
(I-H(\theta)) X & Z
\end{array}\right) \geq 0, \quad \forall \theta \in\left[\theta_{\min }, \theta_{\max }\right], \\
(1-\epsilon)\left(A X+X A^{T}+b y+y^{T} b^{T}\right)+L_{2}+\delta I \leq 0, \\
L_{1}>0, \quad L_{2} \geq \alpha X
\end{gathered}
$$

for some $\mu \in(0,1], \epsilon \in(0,1), \beta_{1} \in(0,1), \alpha, \gamma, \nu, \delta, \theta_{\min }, \theta_{\max } \in \mathbb{R}_{+}, X \in \mathbb{R}^{n \times n}, y \in \mathbb{R}^{1 \times n}$;

2) there exists a $c_{u} \in \mathbb{R}_{+}$that satisfies the inequality (15);

3 ) the disturbance function $d(t, x)$ satisfies the following inequality

$$
d^{T}(t, x) D^{2}\left(\left(c_{1}\|x\|_{r}\right)^{-1}\right) d(t, x) \leq \beta_{2} \delta \alpha\left(c_{2}\|x\|_{r}\right)^{-2 \mu},
$$

where $\beta_{2} \in(0,1)$.

Then the control of the form (16) stabilizes the origin of the system (1) in a finite time and the settling time function is bounded as follows

$$
T\left(x_{0}\right) \leq \frac{c_{2}^{\mu}\left\|x_{0}\right\|_{r}^{\mu}}{\nu \mu\left(2-\beta_{1}-\beta_{2}\right)} .
$$

The proof of the theorem is omitted since it repeats the arguments given for Proposition 1. The numeric procedure presented in Proposition 2 can also be adapted to the conditions of Theorem 5 .

The inequality (40), that restricts the system disturbances, is presented in not appropriate form for practice. Since the inequality (40) is explicitly rewritten form of (20), then we can use the result of Proposition 16 in [9]: 


\section{Proposition 3}

Let $X \in \mathbb{R}^{n \times n}$ be a solution of the system (12), (13), (36)-(39) with $P=X^{-1}$. If

$$
d_{i}^{2}(t, x) \leq \alpha \delta \beta_{2_{i}}\left\{\begin{array}{lll}
\left(\lambda_{\min }(P) x^{T} x\right)^{1+(n-i-1) \mu} & \text { if } \quad x^{T} P x \leq 1, \\
\left(\lambda_{\min }(P) x^{T} x\right)^{\frac{1+(n-i-1) \mu}{1+(n-1) \mu}} & \text { if } \quad & x^{T} P x>1,
\end{array}\right.
$$

for some $\beta_{2_{i}} \in \mathbb{R}_{+}: \beta_{2}=\beta_{2_{1}}+\ldots+\beta_{2_{n}}<1$ then the inequality (40) holds.

In the next section, it is shown by numerical simulation that for obtained control the convergence rate may be slower compared to the result of [13]. To accelerate the convergence rate one can refer to results of the papers [23], [24], [25]. The following corollary is proved in a way similar to [25].

Corollary 2

For $\lambda \geq 1$ and $N=\operatorname{diag}\left\{\lambda^{1-i}\right\}_{i=1}^{n}$ the control

$$
u_{\lambda}(x)=\lambda^{n}\left(c\|N x\|_{r}\right)^{1-\mu} k D\left(\left(c\|N x\|_{r}\right)^{-1}\right) N x,
$$

stabilizes the system (1) in a finite time with the settling time function not exceeding $T \frac{\bar{c}_{2}^{\mu}}{c_{2}^{\mu} \lambda}$, where $c_{2}$ corresponds to (33) and

$$
\bar{c}_{2}=\max \left\{\left(n \lambda_{\max }(\bar{P})\right)^{\frac{1}{2 r_{\max }}},\left(n \lambda_{\max }(\bar{P})\right)^{\frac{1}{2 r_{\min }}}\right\}
$$

for $\bar{P}=N P N$.

\section{Proof of Corollary 2}

Consider the system (1) with the control $u_{\lambda}(x)$ for $d(t, x)=0$

$$
\begin{aligned}
& \dot{x}=A x+b u_{\lambda}(x)=A N^{-1} N x+\lambda^{n} b\left(c\|N x\|_{r}\right)^{1-\mu} k D\left(\left(c\|N x\|_{r}\right)^{-1}\right) N x= \\
& \lambda N^{-1} A N x+\lambda N^{-1} b\left(c\|N x\|_{r}\right)^{1-\mu} k D\left(\left(c\|N x\|_{r}\right)^{-1}\right) N x
\end{aligned}
$$

then for $z=N x$ we obtain

$$
\dot{z}=\lambda[A z+b u(z)] .
$$

The system (44) is finite-time stable since $Z_{\lambda}\left(t, z_{0}\right)=Z\left(\lambda t, z_{0}\right)$, where $Z_{\lambda}\left(t, z_{0}\right)$ is a solution of (44) and $Z\left(\lambda t, z_{0}\right)$ is a solution of the system $\dot{z}=A z+b u(z)$. Then the settling time function is bounded by $T_{z} \leq \frac{V_{z 0}^{\mu}}{\nu \mu(1-\beta)} \lambda^{-1}$, where $V_{z}$ corresponds to the following equation

$$
z^{T} D\left(V_{z}^{-1}\right) P D\left(V_{z}^{-1}\right) z=1 .
$$

Rewrite this equation as $x^{T} D\left(V_{z}^{-1}\right) \bar{P} D\left(V_{z}^{-1}\right) x=1$ and obtain the estimation (43) for the inequality $V_{z} \leq \bar{c}_{2}\|x\|_{r}$

Thus, the settling time function is bounded by $T_{z} \leq \frac{V_{z 0}^{\mu}}{\nu \mu(1-\beta)} \lambda^{-1} \leq \frac{\bar{c}_{2}^{\mu}\left\|x_{0}\right\|_{r}^{\mu}}{\nu \mu(1-\beta)} \lambda^{-1}=T \frac{\bar{c}_{2}^{\mu}}{c_{2}^{\mu} \lambda}$.

The same result can be obtained for the case $d(t, x) \neq 0$.

\section{NUMERICAL EXAMPLE}

Consider the system (1) for $n=3$ in the disturbance free case $(d(t, x)=0)$. By applying Algorithm 1 for $\mu=0.2, \rho=2(1+(n-1) \mu)=2.8, \gamma=1, \nu=0.0035, \theta_{\min }=0.19$ and $\theta_{\max }=2.1$ we 
obtain the next values of the matrix $P \in \mathbb{R}^{3 \times 3}$ and the vector $k \in \mathbb{R}^{1 \times 3}$

$$
\begin{gathered}
P=\left(\begin{array}{lll}
0.0431 & 0.1337 & 0.1437 \\
0.1337 & 0.5822 & 0.6116 \\
0.1437 & 0.6116 & 1.7141
\end{array}\right), \\
k=\left(\begin{array}{lll}
-0.1103 & -0.5461 & -0.9999
\end{array}\right) .
\end{gathered}
$$

The solution of the system (11)-(13), (24), (25) was performed using the MATLAB and YALMIP software with $N=32$ and sampling step $\left(\theta_{i}-\theta_{i-1}\right)=0.0597, i=1, \ldots, N$.

Taking into account estimations of the coefficients $c_{1}$ and $c_{2}$ (34), (35) we can select $c_{1}=0.2604$, $c_{2}=2.6455$ and coefficient $c_{u}$ due to (15) should satisfy the following expression

$$
1.3703 \geq c_{u} \geq 1.2598
$$

Numerical simulations have been done using the coefficient $c_{u}=1.27$ and following initial conditions $x_{1}(0)=5, x_{2}(0)=-6, x_{3}(0)=2$. The simulation results are shown in Fig. 1, Fig. 2.

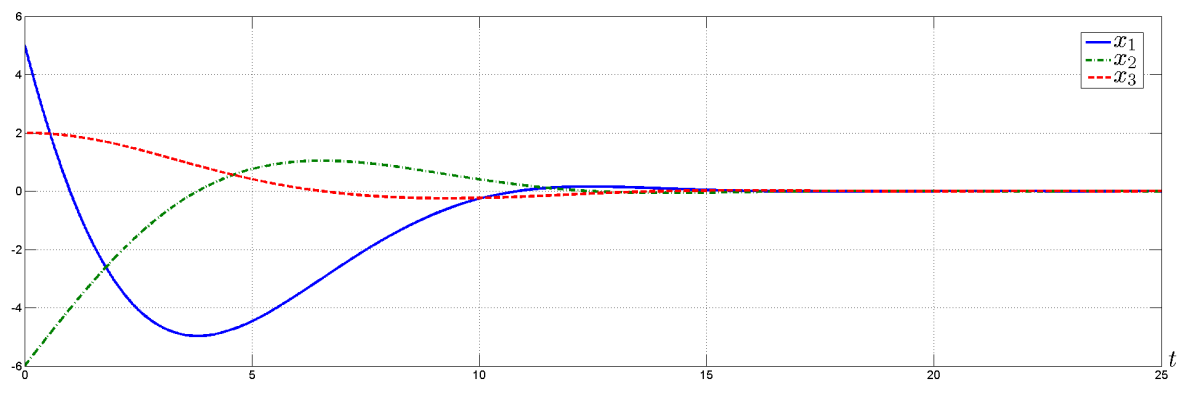

Figure 1. Transients of the system states for the finite-time control

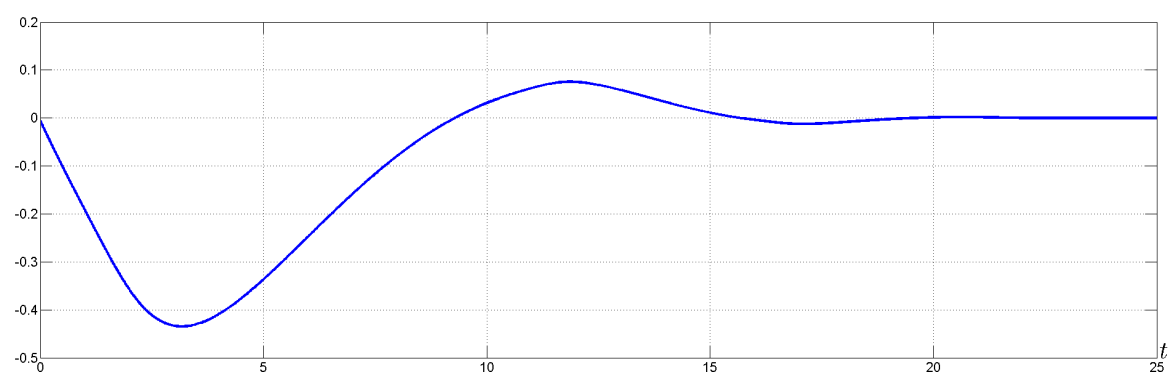

Figure 2. The system input $u$

In order to compare the obtained results with control algorithm (19) presented in [13] the corresponding numerical simulations have been done using the same values of $x_{0}, P, k, \mu$. The simulations results are shown in Fig. 3, Fig. 4.

The simulations results with the control (42) for the same parameter set and $\lambda=5$ are shown in Fig. 5, Fig. 6. The convergence rate is accelerated by $\frac{c_{2}^{\mu} \lambda}{\bar{c}_{2}^{\mu}}=6.8743$ times. 


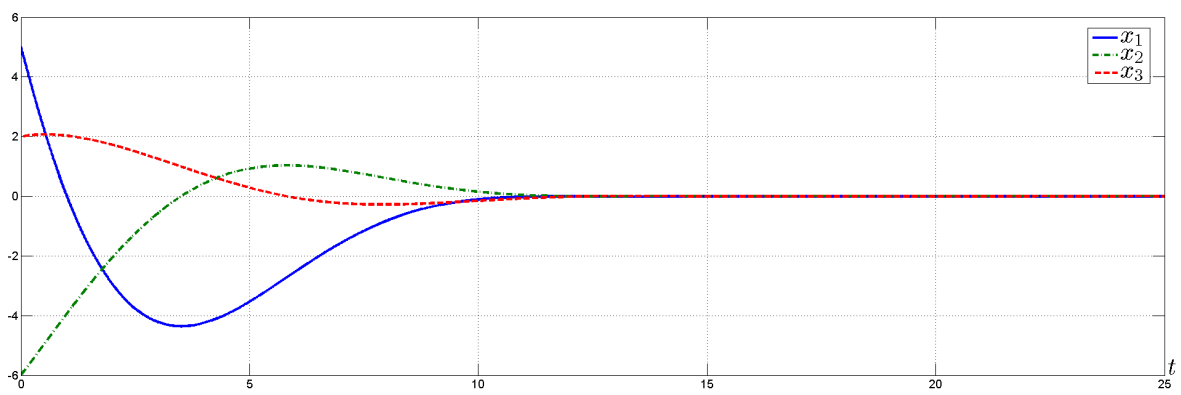

Figure 3. Transients of the system states for the finite-time control (19)

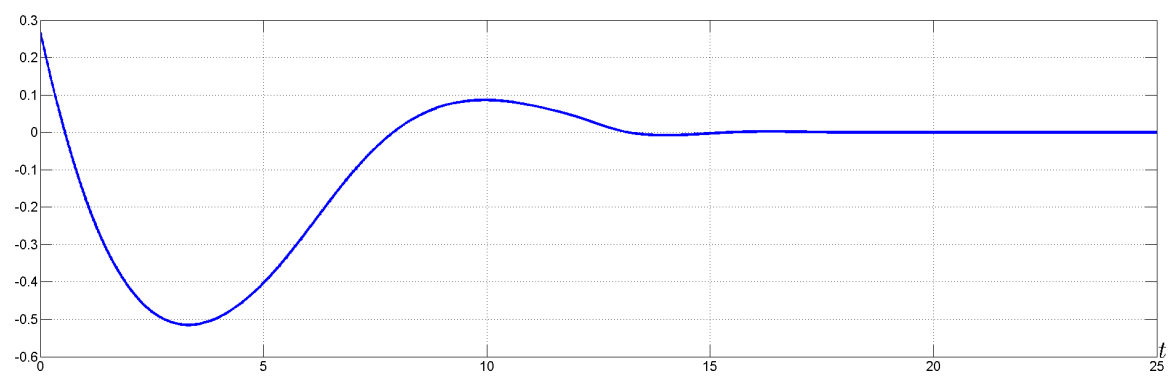

Figure 4. The system input $u$ for the finite-time control presented in [13]

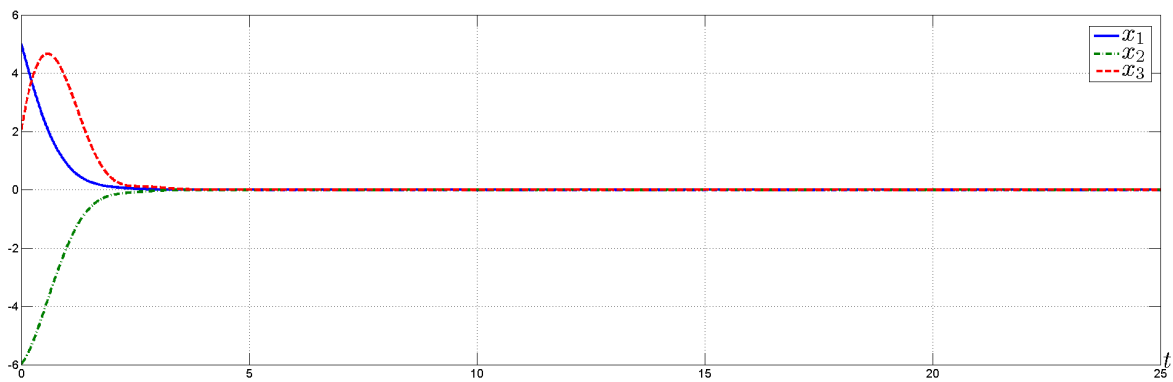

Figure 5. Transients of the system states for the finite-time control (42)

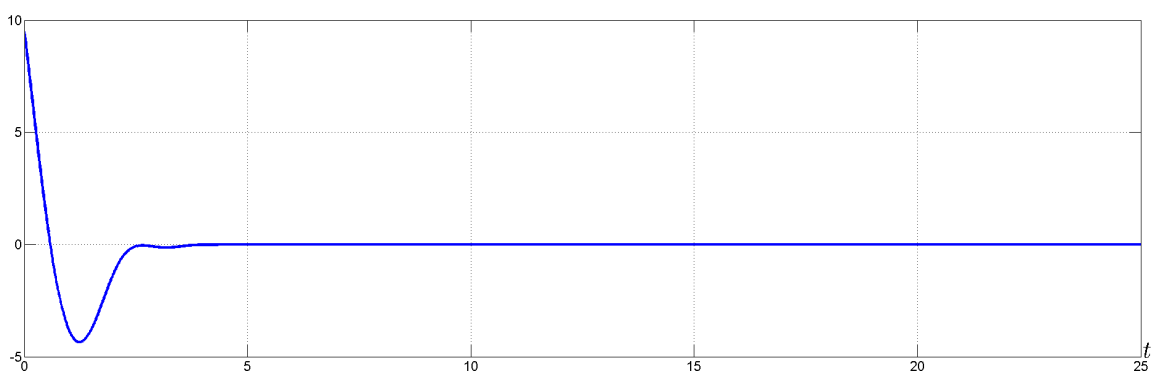

Figure 6. The system input $u$ for accelerated control in the form (42)

Consider the system (1) for $n=3$ in the case of matched disturbances with $d_{1}(t, x)=d_{2}(t, x)=$ $0, d_{3}(t, x)=0.25 \sin \left(\left|x_{1}\right|^{0.5}\right)$. The parameters of the control (16) were selected solving the LMI 
system (12), (13), (36)-(39) for $\mu=0.1, \rho=2(1+(n-1) \mu)=2.4, \epsilon=0.64, \delta=15.5, \alpha=0.33$, $\gamma=5.6, \nu=0.0644, \theta_{\min }=0.19, \theta_{\max }=2.1, N=32$ and $\left(\theta_{i}-\theta_{i-1}\right)=0.0597, i=1, \ldots, N$ :

$$
\begin{gathered}
P=\left(\begin{array}{lll}
0.4367 & 0.3588 & 0.1016 \\
0.3588 & 0.4322 & 0.1195 \\
0.1016 & 0.1195 & 0.0896
\end{array}\right), \\
k=\left(\begin{array}{lll}
-5.5319 & -7.5081 & -3.7437
\end{array}\right) .
\end{gathered}
$$

It is easy to check the disturbance function $d(t, x)$ satisfies the inequality (41). For $c_{1}=0.2566$ and $c_{2}=2.6309$ the parameter $c_{u}$ should satisfy

$$
1.3506 \geq c_{u} \geq 1.2528
$$

The simulation results with $c_{u}=1.3$ are shown in Fig. 7, Fig. 8. The simulation results for accelerated convergence rate with $\lambda=5$ are shown in Fig. 9, Fig. 10.

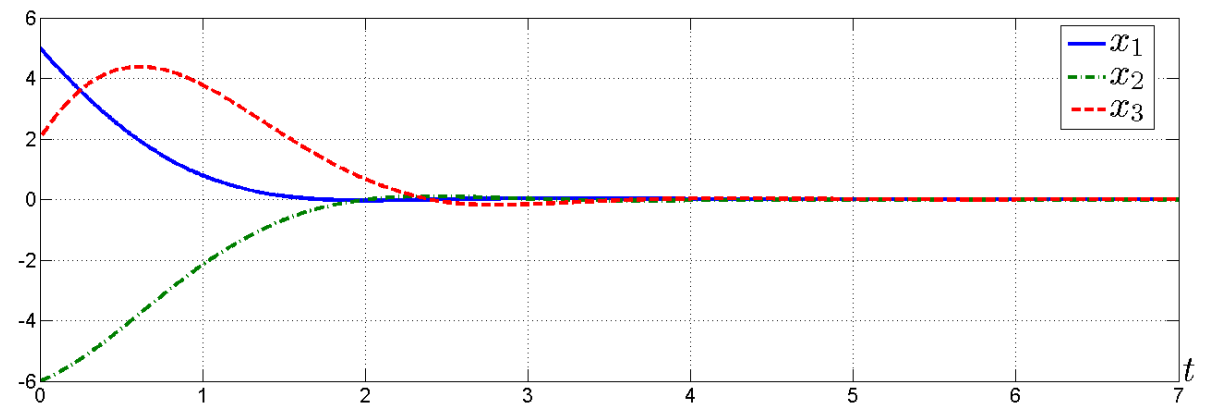

Figure 7. Transients of the system states for the finite-time control

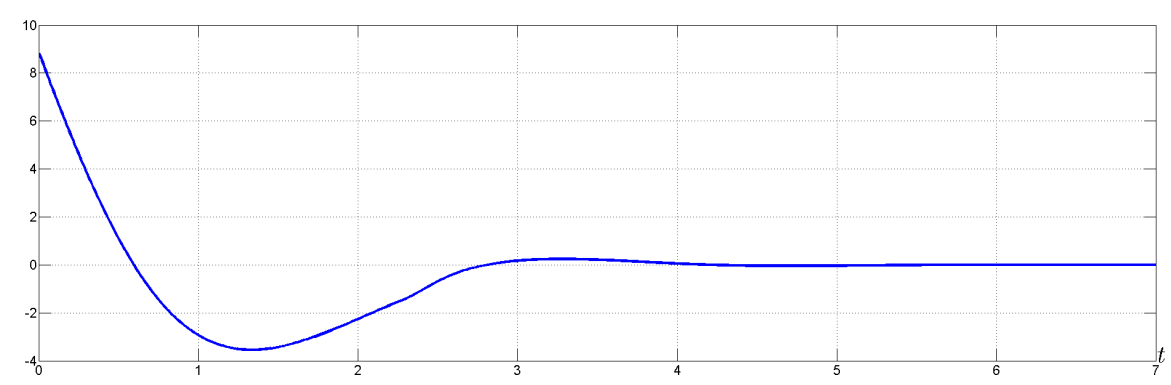

Figure 8 . The system input $u$

\section{CONCLUSION}

The paper presents control algorithm for finite-time stabilization of the system (1). The control design procedure is based on application of the ILF method and some properties of homogeneous 


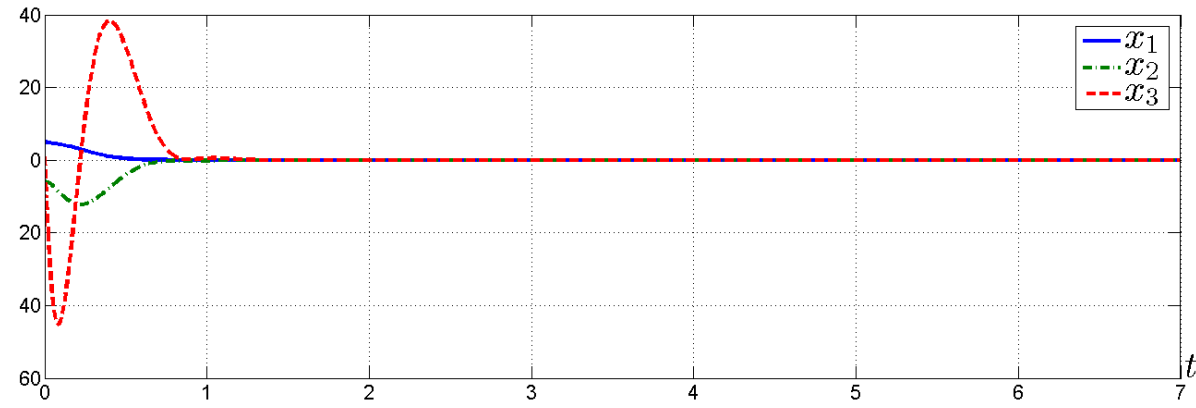

Figure 9. Transients of the system states for the finite-time control (42)

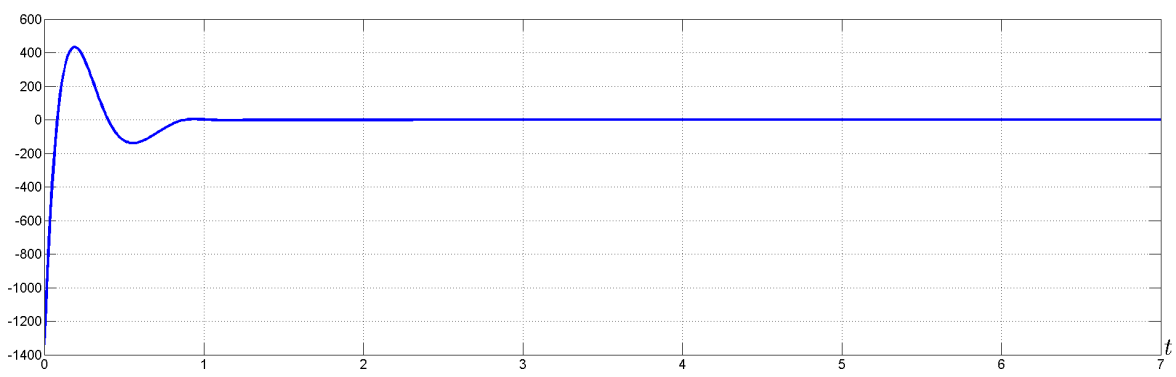

Figure 10. The system input $u$ for accelerated control in the form (42)

systems. Tuning of control parameters is presented in the form of linear matrix inequalities. Numerical example demonstrates the effectiveness of proposed control.

This work is an extension of the paper [13] and in comparison with it the obtained control $u$ does not require on-line procedure for computation of the ILF value at the current state. This issue allows the practical realization of the control scheme to be simplified.

There are many possible directions for further research. For instance, studying robustness with respect to delays or extension of the present result for MIMO and nonlinear systems, extension to the case of output stabilization based on using of finite-time observers (for example, [7], [22]), etc.

\section{REFERENCES}

1. K. Zimenko, A. Polyakov, D. Efimov, Stabilization of Chain of Integrators with Arbitrary Order in Finite-time. In 54th IEEE Conference on Decision and Control, Osaka, Japan, 2015.

2. S.P. Bhat, D.S. Bernstein, Finite-time stability of continuous autonomous systems. SIAM Journal of Control and Optimization, vol. 38(3), pp. 751-766, 2000.

3. E. Roxin, On finite stability in control systems. Rendiconti del Circolo Matematico di Palermo, vol. 15(3), pp. 273-283, 1966.

4. V. Haimo, Finite time controllers. SIAM Journal of Control and Optimization, vol. 24(4), pp. 760-770, 1986.

5. E. Moulay and W. Perruquetti, Finite-time stability and stabilization: State of the art. Lecture Notes in Control and Information Sciences, vol. 334, pp. 23-41, 2006.

6. R. Engel and G. Kreisselmeier, A continuous-time observer which converges in finite time. IEEE Transactions on Automatic Control, vol. 47, pp. 1202-1204, 2002.

7. W. Perruquetti, T. Floquet, and E. Moulay, Finite-time observers: Application to secure communication. IEEE Transactions on Automatic Control, vol. 53(1), pp. 356-360, 2008. 
8. Y. Shen, Y. Huang, and J. Gu, Global finite-time observers for lipschitz nonlinear systems. IEEE Transactions on Automatic Control, vol. 56(2), pp. 418-424, 2011.

9. A. Polyakov, D. Efimov, W. Perruquetti, Robust Stabilization of MIMO Systems in Finite/Fixed Time. Int. J. Robust. Nonlinear Control, vol. 26(1), pp. 69-90, 2016.

10. A. Levant, Homogeneity approach to high-order sliding mode design. Automatica, vol. 41, pp. 823-830, 2005.

11. E. Bernuau, W. Perruquetti, D. Efimov, E. Moulay, Finite-time output stabilization of the double integrator. In Proc. 51st IEEE Conference on Decision and Control, Maui, USA, pp. 5906-5911, 2012.

12. A. Polyakov, L. Fridman, Stability notions and Lyapunov functions for sliding mode control systems. Journal of the Franklin Institute, vol. 351(4), pp. 1831-1865, 2014.

13. A. Polyakov, D. Efimov, W. Perruquetti, Finite-time and fixed-time stabilization: Implicit Lyapunov function approach. Automatica, vol. 51, pp. 332-340, 2015.

14. A.F. Filippov, Differential equations with discontinuous right-hand sides. Kluwer, Dordrecht, 1988.

15. V.I. Zubov, Methods of A.M. Lyapunov and Their Applications. $\quad$ Noordhoff Ltd, Groningen, 263 p., 1964.

16. H. Hermes, Nilpotent approximations of control systems and distributions. SIAM Journal of Control and Optimization, vol. 24, pp. 731736, 1986.

17. V.I. Zubov, On systems of ordinary differential equations with generalized homogenous right-hand sides. Izvestia vuzov. Mathematica, vol. 1, pp. 8088, 1958 (in Russian).

18. A. Bacciotti, L. Rosier, Lyapunov Functions and Stability in Control Theory. S Springer, 237 p., 2005.

19. J. Adamy, A. Flemming, Soft variable-structure controls: a survey. Automatica, vol. 40, pp. 1821-1844, 2004.

20. V.I. Korobov, A general approach to synthesis problem. Doklady Academii Nauk SSSR, vol. 248, pp. 1051-1063, 1979.

21. E. Bernuau, A. Polyakov, D. Efimov, and W. Perruquetti, Verification of ISS, iISS and IOSS properties applying weighted homogeneity. Systems \& Control Letters, vol. 62(12), pp. 1159-1167, 2013.

22. Y. Shen, X. Xia, Semi-global finite-time observers for nonlinear systems. $\quad$ Automatica, vol. 44, no. 12, pp. 31523156, 2008.

23. A. Levant, Finite-time stabilization of uncertain MIMO systems. 53rd IEEE Conference on Decision and Control, pp. 4753-4758, 2014.

24. A. Levant, Y. Dvir, Accelerated high-order MIMO sliding mode control. In Proc. of the 13th International Workshop on Variable Structure Systems, pp. 1-6, 2014.

25. D. Efimov, A. Levant, A. Polyakov, W. Perruquetti, Supervisory Acceleration of Convergence for Homogeneous Systems. International Journal of Control, 2017.

26. Y. Orlov, Finite Time Stability and Robust Control Synthesis of Uncertain Switched Systems. SIAM Journal of Control and Optimization, vol. 43(4), pp. 1253-1271, 2004.

27. Y. Orlov, Discontinuous systems: Lyapunov analysis and robust synthesis under uncertainty conditions. Springer, 320 p., 2009.

28. A. Isidori, Nonlinear Control Systems II. Springer-Verlag London, 293 p., 1999.

29. E. Bernuau, D. Efimov, W. Perruquetti, A. Polyakov, On homogeneity and its application in sliding mode control. Journal of the Franklin Institute, vol. 351(4), pp. 1866-1901, 2014.

30. E. Bernuau, D. Efimov, W. Perruquetti, A. Polyakov, On an Extension of Homogeneity Notion for Differential Inclusions. European Control Conference (ECC), pp. 2204-2209, 2013.

31. R. Courant, F. John, Introduction to calculus and analysis. Vol. II/1, New York: Springer, 2000. 